http://dx.doi.org/10.4314/ajid.v7i1.1

\title{
ANTIBIOTIC RESISTANCE AND VIRULENCE PROPERTIES IN ESCHERICHIA COLI STRAINS FROM CASES OF URINARY TRACT INFECTIONS
}

\section{Felix Oluwasola. Olorunmola ${ }^{1^{*}}$ Deboye Oriade Kolawole $^{2}$ and Adebayo Lamikanra ${ }^{3}$}

\author{
${ }^{1}$ Drug Research and Production, Faculty of Pharmacy, Obafemi Awolowo University, Ile-Ife, \\ Nigeria. \\ *E-mail: folorunmola@gmail.com \\ ${ }^{2}$ Department of Microbiology, Faculty of Science, Obafemi Awolowo University, \\ Ile-Ife, Nigeria. \\ E-mail: dokolawole@yahoo.com \\ ${ }^{3}$ Department of Pharmaceutics, Faculty of Pharmacy, Obafemi Awolowo University, Ile-Ife, \\ Nigeria. \\ E-mail: alamikanra@yahoo.com
}

\begin{abstract}
This study determined E. coli resistance to commonly used antibiotics together with their virulence properties in lle-lfe, Nigeria. A total of $137 \mathrm{E}$. coli isolates from cases of urinary tract infection were tested for their sensitivity to commonly used antibiotics and possession of virulence factors using standard methods. Their ability to transfer resistance was also determined. The isolates demonstrated a high and widespread resistance $(51.1 \%$ to $94.3 \%)$ to all the antibiotics used except Nitrofurantoin $(7.3 \%)$. A total of $50(36.5 \%)$ of the isolates were resistant to 10 of the eleven antibiotics employed. Sixty three per cent (63\%) of the 107 trimethoprim resistant $E$. coli transferred their resistances while amoxicillin, gentamycin, augmentin, tetracycline and erythromycin were cotransferred with trimethoprim. Fifty one (37.2\%) of these multi-resistant isolates possessed one or more virulent factors. The study concluded that urinary tract infection due to $E$. coli in lle-lfe may be difficult to treat empirically except with nitrofurantoin, due to high resistance to commonly used antibiotics. It is imperative that culture and susceptibility tests be carried out on infecting pathogen prior to treatment, in order to avoid treatment failure and reduce selective pressure that could result in the spread of uropathogenic $E$. coli in the environment.
\end{abstract}

Key words: Urinary tract infection, Escherichia coli, antibiotic resistance, virulence factors.

\section{Introduction}

Urinary tract infections are among the most common bacterial infections in humans and Escherichia coli is by far the most commonly recovered bacterial pathogen from both hospital and community patients with this infection (Hryniewcz et. al., 2001, Dasgupta et. al., 2005). The E. coli that cause urinary tract infection are not all the strains from the intestinal tract but a subgroup selected by factors enhancing extra intestinal survival. Such factors include motility by the aid of flagella, structural features such as fimbriae or pili and chemical adhesion (Emody et. al., 2003). The type-1 pili also known to be the most widely distributed among uropathogenic E. coli binds to mannose containing glycoprotein receptors on facet cells lining the bladder or vaginal epithelial cells and are known to be associated with increased severity of UTI (Venegas et. al., 1995; Todar, 2008). An array of other virulence factors has also been identified (Emody et. al., 2003) which confer on some faecal E. coli the ability to colonise the vaginal mucosa and cause symptomatic urinary diseases. This group of $E$. coli is known collectively as uropathogenic E. coli (UPEC) clones (Orskov and Orskov, 1985).

There is the need to eliminate these organisms in order to avoid treatment failure which could lead to proliferation of virulent $E$. coli and consequent morbidity. In settings where resistance to cotrimoxazole (trimethoprim/sulphamethaxole) in $E$. coli is less than $20 \%$, a short course of cotrimoxazole is the recommended empirical treatment for acute uncomplicated cystitis (Lescure et. al., 2001). However the ability of $E$. coli to cause urinary tract infections is on the increase, while the ease of treating these infections due to multidrug antibiotic resistance to first line antibiotics such as cotrimoxazole, ampicillin and nitrofuranton is becoming increasingly elusive. Of greater concern is the recent increase in resistance to fluoroquinolones such as ciprofloxacin and levofloxacin (Hooton, 2003; Karlowsky et. al., 2006).

The increasing ability of $E$. coli to cause urinary tract infections and the difficulty encountered in treating these infections due to multidrug antibiotic resistance necessitates updating the knowledge of their drug resistance in a given environment. This is particularly necessary in a suburban community like lle-lfe of Southwest Nigeria where all kinds of antibiotics are available across the counter with or without prescription (Okeke and Lamikanra, 2001). Hence, the objective of this work was to study and determine the antibiotic susceptibility and resistance of pathogenic $E$. coli isolated from cases of urinary tract infection in Ile-lfe, Nigeria. This is with a view 
http://dx.doi.org/10.4314/ajid.v7i1.1

to selecting the right antibiotics for effective treatment of urinary tract infections caused by Escherichia coli. The study will also demonstrate the virulence factors that contribute to their persistence in UTI patients.

\section{Materials and Methods Isolation of Escherichia coli}

A total of 137 strains of $E$. coli were isolated from urinary tract infections at two tertiary care hospitals in Ile-Ife between May 2003 and December 2005. Clean voided midstream and suprapubic aspirates urine samples were collected daily with the assistance of hospital medical personnel into sterile universal bottles from both inpatients and out-patients suspected to have a urinary tract infection. Culture was done by the calibrated loop technique delivering approximately $0.01 \mathrm{ml}$. of urine, and cultured on MacConkey agar (Oxoid, England) or Cystine-Lactose Electrolyte-Deficient (CLED) agar medium to obtain isolated colonies. Only E.coli colonies showing a significant growth of $10^{5}$ colony forming units per milliliter or more of urine samples after incubation at $37^{\circ} \mathrm{C}$ for $24 \mathrm{~h}$ were considered in this study.

This was based on colony morphology and cultural characteristics on MacConkey agar and EMB agar. The presence or absence of growth, colour, whether they were mucoid or non-mucoid and consistency of colonies produced by each isolate on the media were observed and recorded. The isolates were further identified using standard microbiological methods (Barrow and Feltham, 1993). All Gram negative isolates that showed positive results for motility, lactose fermentation, indole production, and inability to utilize citrate as a sole carbon source were defined as E. coli and stored in Nutrient Agar.

\section{Antimicrobial susceptibility testing}

Isolates were tested for antimicrobial susceptibility by using the standard agar disc diffusion method (NCCLS,1993) on Tryptone soy agar surface already seeded with the organism using cotton tipped applicator (Sterilin Ltd. Middlesex, U.K.). The multidisc used (AB Biodisk) contained the following antibiotics arranged equidistant to each other - amoxicillin, $250 \mu \mathrm{g}$; trimethoprian/sulphamethaxole, $25 \mu \mathrm{g}$; nitrofurantoin, $300 \mu \mathrm{g}$; gentamycin, $10 \mu \mathrm{g}$; nalidixic acid, $30 \mu \mathrm{g}$; ofloxacin, $30 \mu \mathrm{g}$; augmentin, $30 \mu \mathrm{g}$; tetracycline, $30 \mu \mathrm{g}$; erythromycin, 15 $\mu \mathrm{g}$; norfloxacin, $10 \mu \mathrm{g}$; and ciprofloxacin, $10 \mu \mathrm{g}$; The plates were refrigerated at $4{ }^{\circ} \mathrm{C}$ for 30 minutes to ensure adequate diffusion of the antibiotics before incubation at $37{ }^{\circ} \mathrm{C}$ for $18 \mathrm{~h}$. The diameters of inhibition zones were measured in millimeter, and interpreted in accordance with the manufacturer's recommendations (AB Biodisk; PDM Interpretative Chart).

\section{Minimum Inhibitory Concentration (MIC) of Trimethoprim}

The MIC for all trimethoprim resistant $E$. coli isolates was determined by the agar dilution method (NCCLS,1993). A 1:1000 dilution of overnight culture of each isolate was prepared in sterile distilled water and the resultant suspension was applied to the surface of dried iso sensitest agar containing doubling dilution (1024 $4 \mathrm{mg} / \mathrm{l}$ ) of trimethoprim lactate (Welcome Foundation, Sweden) in duplicate plates, with a multipoint inoculators. The concentration of trimethoprim inhibiting growth after $24 \mathrm{~h}$ incubation at $37^{\circ} \mathrm{C}$ was taken as the MIC.

\section{In-vitro Transconjugation experiment}

Mating experiment were conducted for all Trimethoprim resistant isolates on Triptone agar plate with the trimethoprim sensitive, nalidixic acid resistant E. coli K-12 C600 as recipient. Transconjugants were harvested on Tryptone Soy Agar plates containing $10 \mathrm{mg} / \mathrm{L}$ trimethoprim and $40 \mathrm{mg} / \mathrm{L}$ nalidixic acid (Sigma Chemical Company, St. Louis, USA). All transconjugants were screened for threonine marker of $E$. coli K-12 C600 and for resistance to antibiotics to which the donor organisms were resistant.

\section{Detection of virulence factors Capsule formation}

This was done by the combined positive and negative staining method described by Okeke and Lamikanra, [1995]. The organisms appeared red against a blue background, while the presence of capsule was observed as a clear zone around each bacterium.

\section{Haemolysin}

All the E. coli isolates were screened for haemolytic activity on nutrient agar plates containing $7 \%$ washed human erythrocytes. A distinct colony of the organism was streaked and stabbed on the nutrient agar plate containing $7 \%$ washed erythrocyte and incubated at $37^{\circ} \mathrm{C}$ for $18 \mathrm{~h}$. The erythrocytes were then observed for lyses, as indicated by the blood free zone surrounding the colony stab. 
http://dx.doi.org/10.4314/ajid.v7i1.1

\section{Colicin production}

This was carried out using the agar overlay method described by Barth et. al. (1978), E. coli K-12 C600 was used as indicator organism. Plates were observed for the formation of inhibition zones around the test organism stabs.

\section{Haemaglutination}

Haemaglutination was detected by clumping of erythrocytes by fimbriae of bacteria in the presence of Dmannose using the method described by Evans et al. (1979). Washed erythrocytes were recovered from freshly collected blood from human type 'A' donor. Strains were classified as showing instantaneous haemaglutination (4+); almost instantaneous (within $60 \mathrm{~s}$ at room temperature (3+); within 5 min on ice (2+); within 30 min on ice $(1+)$; or no haemaglutination (0).

\section{Results}

A total of $137 \mathrm{E}$. coli strains were isolated from the urine samples that met the inclusion criteria of $10^{5}$ colony forming units per millilitre of urine. Those samples without growth or with growth less than $10^{5} \mathrm{cfu} / \mathrm{ml}$ of urine were excluded from this study. Eighty one isolates, representing $59.1 \%$ of the total isolates were recovered from female patients, while $56(40.9 \%)$ were isolated from male patients.

\section{Antibiotic susceptibility studies of $E$. coli}

The results showed widespread resistance (51.1 - $91.2 \%)$ of the isolates to all the antibiotics, except nitrofurantoin with resistance rate of $7.3 \%$ (Figure 1). Among the fluoroquinolones, ofloxacin showed the least resistance $(51.1 \%)$, followed by Ciprofloxacin $(65.7 \%)$ and Norfloxacin $(86.9 \%)$. The result also showed (Figure 2 ) that the $E$. coli isolates were multiply-resistant with the highest percentage of multiresistant isolates skewed toward the highest number of antibiotics. Up to $50(36.5 \%)$ strains were resistant to 10 out of the 11 antibiotics employed. A total number of $20(14.6 \%), 18(14.14 \%)$ and $13(9.5 \%)$ isolates were resistant to 7,8 and 9 antibiotics respectively, while $8(5.8 \%)$ of the isolates were resistant to all the antibiotics employed.

\section{Transfer of resistance}

Out of the 107 trimethoprim resistant E. coli tested, $63.6 \%$ were able to transfer their resistant traits to plasmidless $E$. coli $\mathrm{K} 12 \mathrm{C} 600$. A high percentage of these isolates that transferred their resistances also fall within the highly resistant strains, i.e., those having their MIC above $1000 \mathrm{mg} / \mathrm{ml}$. of trimethoprim. The result also showed that five of the antibiotics: amoxicillin (88.9\%), gentamycin $(20 \%)$, augmentin $(67.5 \%)$, tetracycline $(72.2$ $\%)$ and erythromycin (57.1\%) were co-transferred with trimethoprim.

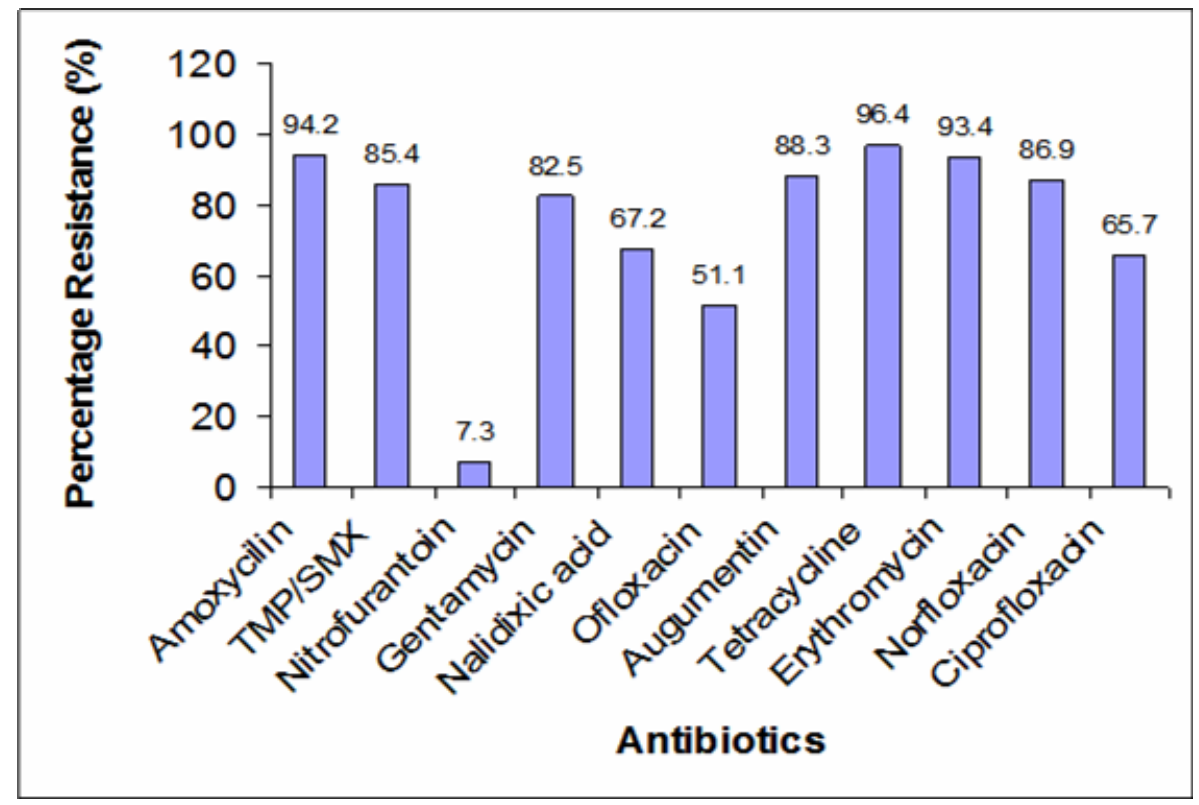

Figure 1: Percentage resistance of E. coli to different antibiotics. 
http://dx.doi.org/10.4314/ajid.v7i1.1

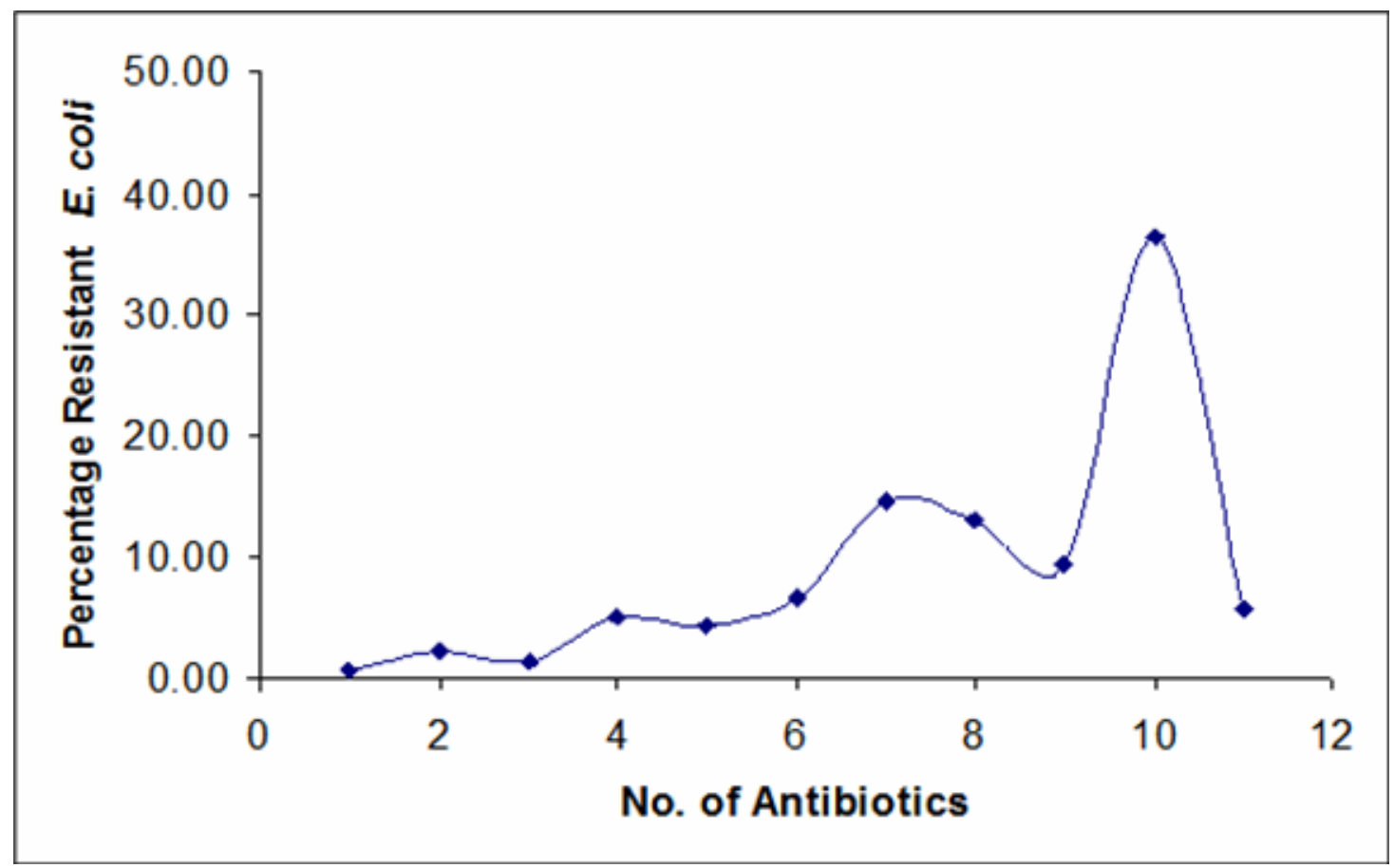

Figure 2: Percentage multiresistance of $E$. coli isolates to the antibiotics.

\section{Occurrence of $E$. coli with virulence markers}

The percentage occurrence of the different virulence markers among the $E$. coli isolates (Table 1) showed that fifty one isolates representing $37.2 \%$ were capsulated. A total of $19(13.9 \%)$ strains showed mannose resistant haemagglutination, while $13(9.5 \%)$ produced the exotoxin colicin. Ten $(7.3 \%)$ isolates were haemolytic. The four different markers also occurred both singly and in different combinations among the isolates and were all multi-resistant (Table 2).

Table 1: Number of Isolates with different Virulence markers

\begin{tabular}{l|l}
\hline Number (\%) of isolates & Virulence property \\
\hline $51(37.2)$ & Capsulated \\
$19(13.9)$ & Mannose resistant haemaglutination \\
$13(9.5)$ & Colicin \\
$10(7.3)$ & Haemolysin \\
\hline
\end{tabular}

\section{Discussion}

The results of this work showed a higher percentage of isolates from female patients $(59.1 \%)$ than males $(40.9 \%)$. This is consistent with previous findings that UTI is more common in females than their male counterparts (Foxman et. al., 2000; Aiyegoro et. al., 2007; Omoregie et. al., 2008). There was a wide range of resistance of the $E$. coli isolates [Figure 1] to all the commonly used antibiotics in this environment, namely, amoxicillin, gentamicin, cotrimoxazole, augmentin, ciprofloxacin, norfloxacin, streptomycin and nalidixic acid. The result also showed a higher level of resistance of $E$. coli than were reported by previous workers (Lamikanra and Ndep, 1989). The result is however in agreement with the report that antibiotic resistance in $E$. coli isolates from urinary tract infection is on the increase (Kurutepe et. al., 2005).

The high level of resistance to the fluoroquinolones limits the options for effective treatment of urinary tract infection caused by $E$. coli in this environment. This is because fluoroquinolone antibiotics were considered to be critical antibiotics due to their very high effectiveness against pathogens that are resistant to other antibiotics. It is evident from this study that fluoroquinolones can no longer be employed as alternative to the less effective trimethoprim/sulphamethaxole (Cotrimoxazole) for empirical treatment of UTI caused by E. coli within this study area. The only exception among the tested antibiotics is nitrofurantoin, to which the organisms exhibited 
http://dx.doi.org/10.4314/ajid.v7i1.1

a resistance as low as $7.3 \%$ [Figure 1]. The high sensitivity shown to this antibiotic is consistent with the result of other workers (Gupta et. al., 2001; Karlosky et. al., 2002), in other parts of the world. The reason while nitrofurantoin continues to show high sensitivity is not yet understood, only that this synthetic antibacterial does not belong to the group of any of the other antibiotics. It may also be related to the fact that the drug is not as commonly used as the other anti-infective agents such that, with less consistent drug pressure, the microbial sensitivity is sustained.

Table 2: Numbers of uropathogenic E. coli isolates with one or more virulence markers and their resistance level.

\begin{tabular}{l|l|l}
\hline Virulence Markers & Number of isolates & Resistant to: \\
\hline One marker & & \\
\hline COL & 8 & $2-10$ antibiotics \\
HL & 2 & $4-10$ antibiotics \\
CAP & 22 & $2-11$ antibiotics \\
MRHA & 10 & $2-11$ antibiotics \\
Total & 42 & \\
Two Markers & & \\
COL+HL & 2 & $9-10$ antibiotics \\
HL+CAP & 3 & $9-10$ antibiotics \\
HL+MRHA & 0 & - \\
CAP+MRHA & 7 & $6-11$ antibiotics \\
COL+CAP & 3 & $7-10$ antibiotics \\
Total & 17 & \\
Three Markers & & 7 antibiotics \\
HL+CAP+MRHA & 1 & \\
Total & 1 &
\end{tabular}

Key: $\mathrm{COL}=$ Colicin formation, $\mathrm{CAP}=$ Capsule, $\mathrm{MRHA}=$ Mannose resistant haemaglutination, $\mathrm{HL}=$ Haemolysin production.

It is evident from the result of this work that majority of the E. coli isolates are highly and multiply-resistant. The reason for this high level of resistance could be due to the fact that ingestion of antibiotics is known to provide selective pressure ultimately leading to a higher prevalence of resistant bacteria (Okeke et. al., 2000). In most towns and cities in Nigeria, these antibiotics are readily made available over the counter without prescriptions. The indiscriminate use and misuse of antibiotics and the consumption of substandard antibiotics as earlier reported (Okeke and Lamikanra, 1995) might have over the years contributed to the high rate of antibiotic resistance recorded in this study.

About $63 \%$ of the trimethoprim resistant isolates were able to transfer their resistance into a plasmidless E. coli strain. It is to be noted also that most of these trimethoprim resistant isolates were able to grow in the presence of more than $1000 \mathrm{mg} / \mathrm{l}$ of trimethoprim, indicative of high level of resistance. Co-transfer of resistance of trimethoprim and amoxicillin was predominant and this was followed by co-transfer with tetracycline, augmentin erythromycin and gentamycin respectively. Adeleye (1992) reported that while genes encoding multiple antibiotic resistances are not always transferable they are often located on easily mobilised plasmids. Therefore, the widespread antibiotic resistance encountered in these isolates can however, be due not only to the indiscriminate use of drugs in this community, but also to the early transferability of plasmids.

Identification and evaluation of virulence factors are necessary for a more complete understanding of the pathogenesis of $E$. coli UTI and may in turn lead to the logical development of new strategies for the prevention and treatment of UTI and other infections caused by the pathogens. In this study, $37.2 \%$ strains were encapsulated (Table 1) and phenotypic characterisation reveals that eight of these encapsulated strains were mucoid within 18-24 $\mathrm{h}$. Capsules are known to confer serum and phagocyte resistance to some E. coli strains (Russo et. al., 1996; Warren, 1997).

Colicin is a narrow spectrum bactericidal macro molecule which is produced by some strains of $E$. coli. Each colicin plasmid is known to confer immunity to the particular colicin which it encodes (Cariksi and Cosar, 2001). Thirteen $(9.5 \%)$ of the 137 E. coli strains in this study produced colicin. Although there have been a dearth of information on colicin production by uropathogenic $E$. coli in this locality, the figure recorded in this study is lower than $25.5 \%, 26.2 \%$ and $36.3 \%$ reported in other parts of the world (Cariksi and Cosar, 2001; Davies et. al., 1981; McGeachie, 1965). This difference may be due to reported differences in the characteristics of strains found in different countries during various periods (Djonne, 1985). Okeke et. al. (2000) reported a high rate of colicinogenicity among enteroaggregative $E$. coli (EAEC) strains isolated from children in Southwest Nigeria and suggested (Okeke et. al., 2000) that this may partially explain the documented association of EAEC with persistent diarrhoea (Bhan et. al., 1989, Baqui et. al., 1992) because abundant adherence and elimination of competing commensals may favour prolonged colonisation and treatment failure. This condition may also be applicable in urinary tract infection. 
http://dx.doi.org/10.4314/ajid.v7i1.1

Agglutination of red blood cells commonly known as haemagglutination is mediated by finbriae (Dugguid et. al., 1979), and is proportionate to the ability to adhere to uroepithelial cells. In mannose resistant haemagglutination (MRHA) the P- blood group antigen acts as a receptor for these organisms and they have improved binding to uroepithelial cells (Emody et. al., 2003). In this study $19(13.9 \%)$ of the isolates produced mannose resistant haemagglutination (Table 1). This value is lower than the $30.9 \%$ reported in India (Raksha et. al., 2003).

Also, a total of ten $(7.3 \%)$ of the isolates produced the toxin, haemolysin. The cytolytic protein toxin secreted by most haemolytic $E$. coli isolates is known as alpha-haemolysin. Three $(30 \%)$ of the ten haemolysin producing $E$. coli were a-haemolytic. Cytotoxic necrotising factor (CNF-1) production increases the capacity of UPEC strains to resist killing by neutrophils, which in turn permits these bacteria to gain access to deeper tissue and persist better in the lower urinary tract (Rippere-Lampe et. al., 2001)

The results also showed that sixty $(53.2 \%)$ of the $E$. coli isolates (Table 2 ) had one or more virulence markers. It has been reported (Raksha et. al., 2003) that haemolysin production, presence of capsule and capacity to cause MRHA emerged as important virulence factors in E. coli UTI. P-fimbriae which often confers on $E$. coli the capacity to cause MRHA is known to induce higher interleukin (IL) -8, IL-6 and neutrophil responses in the urinary tract (Wullt et. al., 2001). In the same way, a-haemolysin, also known as cytotoxic necrotising factor triggers inflammatory response leading to secretion of IL-6 and chemotoxins which set the pace for pathogenesis of renal disease.

The result of this study revealed the occurrence of some virulence factors namely, haemagglutin colicin haemolysin and capsule, which confer pathogenicity on the predominant $E$. coli isolates from urinary tract infections in this environment. It is now evident that a considerable number of $E$. coli isolates from this study community which, apart from being highly and multiply antibiotic resistant, are well equipped to enter the urinary tract and cause symptomatic disease.

It also showed that empirical treatment of $E$. coli UTI is no longer working in this environment. In order to avoid treatment failure, culture and susceptibility tests should be carried out on infecting pathogens before an antibiotic is selected for treatment. This would discourage the indiscriminate use of antibiotics and reduce selective pressure that could result in the spread of virulent and resistant uropathogenic $E$. coli in the environment.

\section{References}

1. Adeleye, A.I. (1992) Conjugal Transferability of Multiple Antibiotic Resistance in three genera of enterobacteriaceae in Nigeria. J Diarrho Dis Res 10(2): 93-96

2. Ayegoro, O. A, Igbinosa, O.O., Ogunmwonyi, I. N., Odjadjare, E. E., Igbinosa, O. E. and Okoh A. I. (2007) Incidence of urinary tract infections (UTI) among children and adolescents in Ile-Ife, Nigeria. Afr J Microbiol Res 1: 13-19

3. Baqui, A. H., Sack, R. B., Black, R. E., Haider, K., Hossain, A., Alim and Yunus, M., Chowdbury, HR and Sidique, A. K. (1992) Enteropathogens associated with acute and persistent diarrhoea in Bangladeshi children less than 5 years of age. J. Infect. Dis. 166, 792 - 796.

4. Barrow, G. and Feltham, R. (1993) Cowan and Steel Manual for the identification of medical bacteria. Cambridge University Press; $\mathrm{p} 331$

5. Barth, P. T., Richards, H. and Data, N. (1978) Copy numbers of existing plasmids in Escherichia coli K-12. J Bact; 135:760-65

6. $\quad$ Bhan, M. K., Bhandari, N., Sazawai, S., Clemens, J., Raj, P., Levine, M. M., and Kaper, J. B. (1989) Descriptive epidemiology of persistent Diarrhoea among young children in rural northern India. Bulletin of the World Health Organisation, 67: $281-288$.

7. Cariksi, A. I. and Cosar, C. (2001) Colicin production and colicin Typing of uropathogenic Escherichia coli. Turk J Med Sc 31: 483-85

8 Dasgupta, P., Sacks, S. H, Khan, M. S. and Sheerin, N. S. (2005) Urinary Tract Infections; $n$ ew insight into a common problem. Postgrad. Med. J. 8183-86(Pubmed).

8. Davies, D. L., Falkiner, F. R and Hardy, K. G. (1981) Colicin V production by clinical isolates of Escherichia coli. Infect. Immun. 31(2), $574-579$.

10. Djonne, B. K. (1985). Colicin production in relation to pathogenicity factors in strains of Escherichia coli isolated from the intestinal tract of piglets. Acta Med. Scand. 26:

145.

11. Dugguid, J. P., Clegg, S. and Wilson, M. I. (1979) The fimbrial and Non-fimbrial haemaglutinins of Escherichia coli. J Med Microbiol 12: 213-28

12. Emody, L., Kerenyi, M. and Nagy, G. (2003) Virulence factors of uropathogenic Escherichia coli. Int. J. Antimicrob Agents 22:529-33

13. Evans, D. J. Jr., Evans, D. G. and DuPont, H. L., (1979) Haemagglutination patterns of enterotoxigenic and enteropathogenic Esherichia coli determined with human, bovine, chicken and guinea pig erythrocytes in the presence and absence of mannose. Infect Immun 23: 336-46

'14. Foxman, B., Barlow, R. D. Arcy, H., Gillespie, B and, Sobel, J. D. (2000) Urinary tract infection; self-reported incidence and associated costs. Ann Epidem 10: 509 - 13. 
http://dx.doi.org/10.4314/ajid.v7i1.1

15. Gupta, K., Sahm, D. F, Mayfield, D. and Stamm, W. E. (2001) Antimicrobial resistance among uropathogens that cause community - acquired urinary tract infections in women: A nationwide analysis. Clin Infect Dis 33: 89-94.

16. Hooton T.M. (2003) Fluoroquinolones and resistance in the treatment of uncomplicated urinary tract infection. Int. J. Antimicrob Agents 2: 65-72.

17. Hryniewics, K., Szczy, K., Sulikowska, A., Jankowski, K., Betlejewska, K. and Hryniewicz W. (2001) Antibiotic Susceptibility of bacterial strains isolated from urinary tract infections in Poland. J. Antimicrob. Chemother. 47: 773 - 80.

18. Karlowsky, J. A., Horban, D. J., DeCorby, M. R., Lang, N. M., and Zhanel, G. G. (2006) Fluoroquinolone Resistant Urinary Isolates of $E$. coli from out patients Are Frequently Multidrug Resistant: Results from the North American Urinary Tract Infection Collaborative Alliance - Quinolone Resistance Study. Antimicrob. Agents Chemother. 50(6) 2251 2254.

19. Karlowsky, J. A., Kelly, L., Thornsberry, C., Jones, M. E. and Sahm, D. F. (2002). Trends in antimicrobial resistance among urinary tract infection isolates of Escherichia coli from female out patients in the United States. Antimicrob Agent Chemother 46(8): 2540-45.

20. Kurutepe, S., Surukuoglu, S., Sezgin, C., Gazi, H., Gulay, M. and Ozbakkaloglu, B. (2005) Increasing antimicrobial resistance in Escherichia coli isolates from community-acquired urinary tract infections during 1998-2003 in manias Turkey. Jap J Inf Dis 58: 159-61.

21. Lamikanra, A. and Ndep, R. B. (1989) Trimethoprim resistance in urinary tract pathogens in two Nigerian hospitals. J Antimicrob Chemo 23:151 - 54.

22. Lescure, F. X., Eveillard, M., Douadi, Y. and Eb, F. (2001) Community -acquired multiresistant bacteria : an emerging problem? J. Hosp. Infect.; 49: 149 - 151.

23. McGeachie, J. (1965) Bacteriocin typing in urinary infection. Zbl. Bact., Abt. (Orig.), 196, 377 - 384.

24. NCCLS. (1993) Performance standards of antimicrobial disk susceptibility tests, 4th Edn. National Committee for Clinical Laboratory Standards, Villanova, PA, Approved Standard M2 - A5.

25. Okeke, I. N., Fayinka, S.T and Lamikanra, A. (2000) Antibiotic resistance in Escherichia coli from Nigerian students, 1986-1998. Emerg Inf Dis 6(4): 393-96

26. Okeke IN, Lamikanra A. (1995) Quality and bioavailability of Tetracycline capsules in a Nigerian semi urban community. Int J Antimicrob Agents 5: 245-50. multiresistant bacteria : an emerging problem? J. Hosp. Infect.; 49: 149 151.

27. Okeke, I. N. and Lamikanra, A. (1995) Bacterial capsules; a simple method for demonstration un,der the light microscope. Brit J Biomed Sc 52: 321 - 22

28. Okeke, I. and Lamikanra A. (2001) Quality and bioavailability of ampicillin capsules dispensed in a Nigerian semiurban community Afr J Med Sc;30:47-51.

29. Okeke, I. N., Lamikanra, A., Czeczulin, Dubovsky, F., Kaper, J. B. and Nataro, J. P. (2000). Heterogenous virulence of Enteroagregative Escherichia coli strains isolated from children in Southwest Nigeria. J. Infect. Dis. 181(1): 252 -260 .

30. $\quad$ Omoregie, R., Erebor, J. O., Ahonkhai, I., Isibor, J. O., Ogefere, H. O. (2008). Observed changes in The prevalence of uropathogens in Benin City, Nigeria NZ J Med Lab Sci 62: 29-31.

31. Orskov and Orskov, (1985). Escherichia coli in extra-intestinal infections. Journal of Hygiene (London); 95: 551-

575

32. Raksha, R., Srinivasa, H. and Macaden, R. S. (2003) Occurrence and characterization of uropathogenic Escherichia coli in urinary tract infections. Ind. J. Med. Microbiol 21(2): 102-107

33. Rippere-Lampe, K. E., O’Brien, A. D., Conran, R., and Lock, H. A., (2001) Mutation of the gene encoding cytotoxic necrotizing factor type 1 [cnf(1)] attenuates the virulence of uropathogenic Escherichia coli. Infect Immun 69(6): $3954-3964$.

34. Russo, T. A., Brown, J. J., Jodush, S. T. and Johnson, J. R. (1996) The O4 Specific Antigen Moiety of Lipopolysaccharide but not the K54 Group 2 Capsule is important for Urovirulence of an extraintestinal isolate of Escherichia coli. Infect Immun 2343-48.

35. Todar's Online Textbook of Bacteriology (TOTB): Urinary tract infection. Available on: http://www.textbookofbacteriology.net/e.coli.htm

36. Venegas, M. F., Navas, E. L., Gaffney, R. A., Duncan, J. L., Anderson, B. E. and Schaeffer, A. J. (1995) Binding of type 1-piliated Escherichia coli to vaginal mucus. Infect and Immun 63(2): 416-22.

37. Warren, J. W. (1997) Host parasite interactions and host defence mechanisms...Eds (Little Brown, London). 873894.

38. Wullt, B., Bergsten, G., Connell, H., Rollano, P., Gebratsedik, N., Hang, L. and Svanborg, C. (2001) P-fimbriae trigger mucosal responses to Escherichia coli in the human urinary tract. Cell Microbiol 3(4): 255-264. 